\title{
Closed Form Solutions of the Perturbed Gerdjikov- Ivanov Equation With Variable Coefficients
}

\author{
C.A. Gómez S. ${ }^{1}$, A. Jhangeer ${ }^{2}$, H. Rezazadeh ${ }^{3}$, R.A. Talarposhti ${ }^{3}$ \\ and A. Bekir ${ }^{4, *}$ \\ ${ }^{1}$ Department of Mathematics, Universidad Nacional de Colombia, Bogotá, \\ Colombia. \\ ${ }^{2}$ Department of Mathematics, Namal Institute, 30KM Talagang Road, \\ Mianwali 42250, Pakistan. \\ ${ }^{3}$ Faculty of Engineering Technology, Amol University of Special Modern \\ Technologies, Amol, Iran. \\ ${ }^{4}$ Neighbourhood of Akcaglan, Imarli Street, Number: 28/4, 26030, \\ Eskisehir, Turkey.
}

Received 23 June 2020; Accepted (in revised version) 7 September 2020.

\begin{abstract}
The solutions of the perturbed Gerdjikov-Ivanov equation with coefficients depending on temporal variable are obtained. They are presented in terms of generalised solitary and periodic solutions and can be used to determine new solutions of the classical perturbed Gerdjikov-Ivanov equation with special sets of constant coefficients.
\end{abstract}

AMS subject classifications: 35R11, 35A20, 35C05

Key words: Perturbed Gerdjikov-Ivanov equation with variable coefficients, periodic and soliton solutions, improved tanh-coth method, Exp-function method.

\section{Introduction}

It is well known that certain solutions of nonlinear partial differential equations provide a better understanding of various phenomena. From the mathematical point of view, the use of generalised models is important in the sense that in particular cases the solutions are derived by changing model parameters. In this work, we use the improved tanh-coth method [20] in order to find exact traveling wave solutions of the following generalised perturbed Gerdjikov-Ivanov equation

$$
\begin{aligned}
i q_{t} & +A(t) q_{x x}+B(t)|q|^{4} q+i \\
& \times\left[C(t) q^{2} q_{x}^{*}+\rho(t) q_{x}+\delta(t)\left(|q|^{2 m} q\right)_{x}+\mu(t)\left(|q|^{2 m}\right)_{x} q\right]=0,
\end{aligned}
$$

*Corresponding author. Email addresses: cagomezsi@unal.edu.com (C.A. Gómez), adil.jhangeer@ gmail.com (A. Jhangeer), h.rezazadeh@ausmt.ac.ir (H. Rezazadeh), talarposhti@yahoo.com (R.A. Talarposhti), bekirahmet@gmail.com (A. Bekir) 
where $q=q(x, t)$ is a complex-valued wave profile depending on spatial and temporal variables $x$ and $t$, and $q^{*}$ denotes the complex conjugate of $q-\operatorname{cf}$. $[9,14,15]$. If the coefficients $A, B, C, \rho, \delta$ and $\mu$ are constants, then (1.1) is the standard perturbed Gerdjikov-Ivanov equation - cf. $[6,21,26]$. In this case, $A$ is the group velocity dispersion coefficient, $B$ the quintic nonlinearity coefficient, $C$ the nonlinear dispersion coefficient, $\rho$ the inter-modal dispersion coefficient, $\delta$ the self-steepening coefficient, $\mu$ the higher-order dispersion coefficient and $\imath:=\sqrt{-1}$. It is worth noting that for non-constant coefficients, the structure of the corresponding solutions of (1.1) differs from that for the standard Gerdjikov-Ivanov equation. For more details the reader is referred to [3, 10-13, 16, 27]. We note that the easily numerically implementable improved tanh-coth method of [20] can be considered as a generalisation of classical methods such as the tanh-coth method [24], the Kudryashov method [8], the $G^{\prime} / G$ method [23], the $\operatorname{Exp}(-\phi(\xi))$ method [4] and the methods considered in $[1,7,17-19,22,25]$.

This paper is organised as follows. In Section 2 we review the improved tanh-coth and Exp-function methods for nonlinear partial differential equations. New exact traveling wave solutions of the Eq. (1.1) are derived in Section 3. Finally, some conclusions are given in Section 4.

\section{Description of the Methods}

Given a nonlinear partial differential equation

$$
P\left(u, u_{x}, u_{t}, u_{x t}, u_{x x}, \ldots\right)=0,
$$

where $u=u(x, t)$ is the unknown function, $x$ the spatial variable, $t$ the temporal variable, and the coefficients of (2.1) depend on the variable $t$ only, transformations similar to the transformation

$$
\xi=x+\lambda t+\xi_{0}
$$

reduce the Eq. 2.1 to an ordinary differential equation

$$
P_{1}\left(u, u^{\prime}, u^{\prime \prime}, \ldots\right)=0
$$

with an unknown function $u=u(\xi)$.

\subsection{An improved tanh-coth method}

We consider a tanh-coth method consisting in finding the solutions of (2.2), which can be represented in the form

$$
u(\xi)=\sum_{i=0}^{M} a_{i}(t) \phi(\xi)^{i}+\sum_{i=M+1}^{2 M} a_{i}(t) \phi(\xi)^{M-i},
$$

where $M$ is a positive integer to be determined later and $\phi=\phi(\xi)$ satisfies the general Riccati equation

$$
\phi^{\prime}(\xi)=\gamma(t) \phi^{2}(\xi)+\beta(t) \phi(\xi)+\alpha(t) .
$$


The solutions of the Eq. (2.4) are known [2], viz.

$$
\phi(\xi)= \begin{cases}\frac{\sqrt{\Pi} \tan \left[\frac{1}{2} \sqrt{-\Pi}\left(\xi+\xi_{0}\right)\right]-\beta(t)}{2 \gamma(t)}, & \Pi<0, \\ \frac{-\sqrt{\Pi} \cot \left[\frac{1}{2} \sqrt{-\Pi}\left(\xi+\xi_{0}\right)\right]-\beta(t)}{2 \gamma(t)}, & \Pi<0, \\ \frac{-\sqrt{\Pi} \tanh \left[\frac{1}{2} \sqrt{\Pi}\left(\xi+\xi_{0}\right)\right]-\beta(t)}{2 \gamma(t)}, & \Pi>0, \\ \frac{-\sqrt{\Pi} \operatorname{coth}\left[\frac{1}{2} \sqrt{\Pi}\left(\xi+\xi_{0}\right)\right]-\beta(t)}{2 \gamma(t)}, & \Pi>0, \\ \frac{1}{\gamma(t)}\left(-\frac{1}{\left(\xi+\xi_{0}\right)}-\frac{\beta(t)}{2}\right), \quad \beta^{2}(t)=4 \alpha(t) \gamma(t), & \end{cases}
$$

where

$$
\Pi=\beta^{2}(t)-4 \alpha(t) \gamma(t)
$$

It is clear that varying the parameter $\xi_{0}$ in (2.5), we can derive various solutions, including the periodic ones, depending of the sign of $\beta^{2}(t)-4 \alpha(t) \gamma(t)$, cf. [2]. Substituting (2.3) into (2.2) and balancing the linear terms of highest order with the highest order nonlinear term we determine $M$. This $M$ is used in (2.3) and substituting the corresponding expression into (2.2) and employing (2.4), we arrive at an algebraic system of equations with respect to variables $\alpha(t), \beta(t), \gamma(t), \lambda(t), a_{0}(t), \ldots, a_{2 M}, b_{0}(t), \ldots, b_{2 N}(t)$. Solving the system and reversing the transformations used, we obtain a set of the exact solutions of (1.1) with periodic and soliton solutions among the others.

\subsection{The Exp-function method}

The Exp-function method is based on the assumption that travelling wave solutions can be in the form

$$
p=\frac{\sum_{n=-c}^{d} a_{n} e^{n \xi}}{\sum_{m=-r}^{s} b_{m} e^{m \xi}}
$$

where $c, d, r, s$ are positive integers to be determined later and $a_{n}, b_{m}$ unknown functions depending on $t[5]$. The Eq. (2.6) can be written as

$$
p(\eta)=\frac{a_{c} e^{c \xi}+\cdots+a_{d} e^{d \xi}}{b_{r} e^{r \xi}+\cdots+b_{s} e^{s \xi}} .
$$

In order to determine the values of $c$ and $r$ in the Eq. (2.6), we balance the linear and nonlinear terms of the highest order. Similar, for $d$ and $s$, we balance the linear and nonlinear terms of the lowest order. 


\section{Exact Solutions of the Eq. (1.1)}

Following the previous considerations, we consider the transformation

$$
\begin{aligned}
& q(x, t)=e^{i\left(x+\int \omega(t) d t+\theta_{0}\right)} u(\xi) \\
& \xi=x+\int \lambda(t) d t+\xi_{0} .
\end{aligned}
$$

Substituting (3.1) into (1.1), using the relations

$$
\begin{aligned}
& i q_{t}=\left(\imath \lambda(t) u^{\prime}(\xi)-\omega(t) u(\xi)\right) e^{i\left(x+\int \omega(t) d t+\theta_{0}\right)}, \\
& q_{x}=\left(u^{\prime}(\xi)+i u(\xi)\right) e^{i\left(x+\int \omega(t) d t+\theta_{0}\right)}, \\
& A(t) q_{x x}=A(t)\left(u^{\prime \prime}(\xi)+2 i u^{\prime}(\xi)-u(\xi)\right) e^{i\left(x+\int \omega(t) d t+\theta_{0}\right)}, \\
& q^{2} q_{x}^{*}=\left(u(\xi)^{2} u^{\prime}(\xi)-i u(\xi)^{3}\right) e^{i\left(x+\int \omega(t) d t+\theta_{0}\right)}, \\
& B(t)|q|^{4} q=B(t) u(\xi)^{5} e^{i\left(x+\int \omega(t) d t+\theta_{0}\right)}, \\
& \delta(t)\left(|q|^{2 m} q\right)_{x}=\delta(t)\left[(2 m+1) u(\xi)^{2 m} u^{\prime}(\xi)+i u(\xi)^{2 m+1}\right] e^{i\left(x+\int \omega(t) d t+\theta_{0}\right)}, \\
& \mu(t)\left(|q|_{x}^{2 m} q\right)=\mu(t)\left(2 m u(\xi)^{2 m} u^{\prime}(\xi)\right) e^{i\left(x+\int \omega(t) d t+\theta_{0}\right)},
\end{aligned}
$$

and separating the real and imaginary parts in the resulting expression, we arrive at the following equations:

$$
\begin{aligned}
& A(t) u^{\prime \prime}(\xi)-(\omega(t)+A(t)+\rho(t)) u(\xi)+C(t) u(\xi)^{3} \\
& \quad-\delta(t) u(\xi)^{2 m+1}+B(t) u(\xi)^{5}=0, \\
& \lambda(t)+2 A(t)+\rho(t)+C(t) u(\xi)^{2}+[(2 m+1) \delta(t)+2 m \mu(t)] u(\xi)^{2 m}=0 .
\end{aligned}
$$

It follows from (3.3) that $C(t)=0$ for $m \neq 1$. In the case $m=1$, (3.3) takes the form

$$
\lambda(t)=-2 A(t)-\rho(t), \quad C(t)+3 \delta(t)+2 \mu(t)=0 .
$$

The variable change

$$
u(\xi)=\sqrt{v(\xi)}
$$

transforms (3.2) into the following equation for $m \neq 1$ :

$$
\begin{aligned}
& 2 A(t) v(\xi) v^{\prime \prime}(\xi)-A(t)(v(\xi))^{\prime}-4(\omega(t)+A(t)+\rho(t)) v(\xi)^{2} \\
& \quad+4 C(t) v(\xi)^{3}-4 \delta(t) v(\xi)^{m+2}+4(t) v(\xi)^{4}=0
\end{aligned}
$$

and if $m=1$, we have:

$$
\begin{aligned}
& 2 A(t) v(\xi) v^{\prime \prime}(\xi)-A(t)(v(\xi))^{\prime}-4(\omega(t)+A(t)+\rho(t)) v(\xi)^{2} \\
& \quad+4[C(t)-\delta(t)] v(\xi)^{3}+4 B(t) v(\xi)^{4}=0
\end{aligned}
$$




\subsection{Application of the improved tanh-coth}

Substituting (2.3) into (3.5) and balancing $v(\xi)^{4}$ with $v(\xi) v^{\prime \prime}(\xi)$ or $v(\xi)^{4}$ with $\left(v^{\prime}(\xi)\right)^{2}$ yields $4 M=2 M+2$, so that $M=1$. Therefore, (2.3) takes the form

$$
u(\xi)=a_{0}(t)+a_{1}(t) \phi(\xi)+a_{2}(t) \phi(\xi)^{-1} .
$$

Substituting (3.6) into (3.5) again, we obtain an algebraic system connecting $\alpha(t), \beta(t)$, $\gamma(t), \omega(t), a_{0}(t), a_{1}(t), a_{2}(t)$, viz.

$$
\begin{aligned}
& 4 a_{1}^{2}(t) A(t) \beta(t) \gamma(t)+4 a_{0}(t) a_{1}(t) A(t) \gamma^{2}(t)+16 a_{0}(t) a_{1}^{3}(t) B(t) \\
& +4 a_{1}^{3}(t) C(t)-4 a_{1}^{3}(t) \delta(t)=0, \\
& 3 a_{1}^{2}(t) A(t) \gamma^{2}(t)+4 a_{1}^{4}(t) B(t)=0, \\
& 4 \alpha(t) a_{1}(t) a_{0}(t) A(t) \gamma(t)+2 a_{1}(t) a_{0}(t) A(t) \beta^{2}(t) \\
& +12 a_{1}(t) a_{2}(t) A(t) \beta(t) \gamma(t)-8 a_{1}(t) a_{0}(t) A(t) \\
& +16 a_{1}(t) a_{0}^{3}(t) B(t)+48 a_{1}^{2}(t) a_{2}(t) a_{0}(t) B(t) \\
& +12 a_{1}(t) a_{0}^{2}(t) C(t)+12 a_{1}^{2}(t) a_{2}(t) C(t) \\
& -12 a_{1}(t) a_{0}^{2}(t) \delta(t)-12 a_{1}^{2}(t) a_{2}(t) \delta(t) \\
& -8 a_{1}(t) a_{0}(t) \rho(t)-8 a_{1}(t) a_{0}(t) \omega(t)=0, \\
& 2 \alpha(t) a_{1}^{2}(t) A(t) \gamma(t)+a_{1}^{2}(t) A(t) \beta^{2}(t)+6 a_{0}(t) a_{1}(t) A(t) \beta(t) \gamma(t) \\
& +6 a_{2}(t) a_{1}(t) A(t) \gamma^{2}(t)-4 a_{1}^{2}(t) A(t)+16 a_{2}(t) a_{1}^{3}(t) B(t) \\
& +24 a_{0}^{2}(t) a_{1}^{2}(t) B(t)+12 a_{0}(t) a_{1}^{2}(t) C(t)-12 a_{0}(t) a_{1}^{2}(t) \delta(t) \\
& -4 a_{1}^{2}(t) \rho(t)-4 a_{1}^{2}(t) \omega(t)=0, \\
& 12 \alpha(t) a_{1}(t) a_{2}(t) A(t) \beta(t)+4 \alpha(t) a_{2}(t) a_{0}(t) A(t) \gamma(t) \\
& +2 a_{2}(t) a_{0}(t) A(t) \beta^{2}(t)-8 a_{2}(t) a_{0}(t) A(t) \\
& +16 a_{2}(t) a_{0}^{3}(t) B(t)+48 a_{1}(t) a_{2}^{2}(t) a_{0}(t) B(t) \\
& +12 a_{2}(t) a_{0}^{2}(t) C(t)+12 a_{1}(t) a_{2}^{2}(t) C(t) \\
& -12 a_{2}(t) a_{0}^{2}(t) \delta(t)-12 a_{1}(t) a_{2}^{2}(t) \delta(t) \\
& -8 a_{2}(t) a_{0}(t) \rho(t)-8 a_{2}(t) a_{0}(t) \omega(t)=0, \\
& -\alpha^{2}(t) a_{1}^{2}(t) A(t)+2 \alpha(t) a_{1}(t) a_{0}(t) A(t) \beta(t) \\
& +12 \alpha(t) a_{1}(t) a_{2}(t) A(t) \gamma(t)+6 a_{1}(t) a_{2}(t) A(t) \beta^{2}(t) \\
& +2 a_{2}(t) a_{0}(t) A(t) \beta(t) \gamma(t)-a_{2}^{2}(t) A(t) \gamma^{2}(t)-4 a_{0}^{2}(t) A(t) \\
& -8 a_{1}(t) a_{2}(t) A(t)+4 a_{0}^{4}(t) B(t)+48 a_{1}(t) a_{2}(t) a_{0}^{2}(t) B(t) \\
& +24 a_{1}^{2}(t) a_{2}^{2}(t) B(t)+4 a_{0}^{3}(t) C(t)+24 a_{1}(t) a_{2}(t) a_{0}(t) C(t) \\
& -4 a_{0}^{3}(t) \delta(t)-24 a_{1}(t) a_{2}(t) a_{0}(t) \delta(t)-4 a_{0}^{2}(t) \rho(t) \\
& -8 a_{1}(t) a_{2} v \rho(t)-4 a_{0}^{2}(t) \omega(t)-8 a_{1}(t) a_{2}(t) \omega(t)=0, \\
& 4 \alpha^{2}(t) a_{0}(t) a_{2}(t) A(t)+4 \alpha(t) a_{2}^{2}(t) A(t) \beta(t)+16 a_{0}(t) a_{2}^{3}(t) B(t)
\end{aligned}
$$




$$
\begin{aligned}
& \quad+4 a_{2}^{3}(t) C(t)-4 a_{2}^{3}(t) \delta(t)=0 \\
& 6 \alpha^{2}(t) a_{1}(t) a_{2}(t) A(t)+6 \alpha(t) a_{0}(t) a_{2}(t) A(t) \beta(t) \\
& \quad+2 \alpha(t) a_{2}^{2}(t) A(t) \gamma(t)+a_{2}^{2}(t) A(t) \beta^{2}(t)-4 a_{2}^{2}(t) A(t) \\
& \quad+16 a_{1}(t) a_{2}^{3}(t) B(t)+24 a_{0}^{2}(t) a_{2}^{2}(t) B(t)+12 a_{0}(t) a_{2}^{2}(t) C(t) \\
& \quad-12 a_{0}(t) a_{2}^{2}(t) \delta(t)-4 a_{2}^{2}(t) \rho(t)-4 a_{2}^{2}(t) \omega(t)=0, \\
& 3 \alpha^{2}(t) a_{2}^{2}(t) A(t)+4 a_{2}^{4}(t) B(t)=0 .
\end{aligned}
$$

We use Mathematica software for solving the previous system, thus obtaining a family of solutions. However, here we consider only the following most general set:

$$
\begin{aligned}
& a_{0}(t)=-\frac{3(C(t)-\delta(t))}{4 B(t)}, \quad \alpha(t)=\frac{2\left(\Phi_{2}\right)}{3(C(t)-\delta(t))} \\
& \beta(t)=-\frac{i \sqrt{3}(C(t)-\delta(t))}{2 A(t)^{1 / 2} B(t)^{1 / 2}}, \quad \gamma(t)=\frac{2\left(\Phi_{1}\right)}{3(C(t)-\delta(t))}, \\
& \omega(t)=\frac{64 a_{1}(t) a_{2}(t) B^{2}(t)-48 A(t) B(t)-48 B(t) \rho(t)-9 C^{2}(t)+18 C(t) \delta(t)-9 \delta^{2}(t)}{48 B(t)},
\end{aligned}
$$

where

$$
\begin{aligned}
& \Phi_{1}=\frac{i \sqrt{3} a_{1}(t) B(t)^{1 / 2} C(t)}{A(t)^{1 / 2}}-\frac{i \sqrt{3} a_{1}(t) B(t)^{1 / 2} \delta(t)}{A(t)^{1 / 2}} \\
& \Phi_{2}=\frac{i \sqrt{3} a_{2}(t) B(t)^{1 / 2} C(t)}{A(t)^{1 / 2}}-\frac{i \sqrt{3} a_{2}(t) B(t)^{1 / 2} \delta(t)}{A(t)^{1 / 2}} .
\end{aligned}
$$

For this coefficient set, the Eq. (2.5) takes the form

$$
\begin{aligned}
& \phi_{1}(\xi)=\frac{3(C(t)-\delta(t))}{4\left(\Phi_{1}\right)}\left(-\frac{\chi^{1 / 2}}{2 \sqrt{3}} \tan \left(\frac{\chi^{1 / 2}}{4 \sqrt{3}}\left(\xi+\xi_{0}\right)\right)+\frac{i \sqrt{3}(C(t)-\delta(t))}{2 A(t)^{1 / 2} B(t)^{1 / 2}}\right) \\
& \phi_{2}(\xi)=\frac{3(C(t)-\delta(t))}{4\left(\Phi_{1}\right)}\left(-\frac{\chi^{1 / 2}}{2 \sqrt{3}} \cot \left(\frac{\chi^{1 / 2}}{4 \sqrt{3}}\left(\xi+\xi_{0}\right)\right)+\frac{i \sqrt{3}(C(t)-\delta(t))}{2 A(t)^{1 / 2} B(t)^{1 / 2}}\right) \\
& \phi_{3}(\xi)=\frac{3(C(t)-\delta(t))}{4\left(\Phi_{1}\right)}\left(-\frac{\chi^{1 / 2}}{2 \sqrt{3}} \tanh \left(\frac{\chi^{1 / 2}}{4 \sqrt{3}}\left(\xi+\xi_{0}\right)\right)+\frac{i \sqrt{3}(C(t)-\delta(t))}{2 A(t)^{1 / 2} B(t)^{1 / 2}}\right) \\
& \phi_{4}(\xi)=\frac{3(C(t)-\delta(t))}{4\left(\Phi_{1}\right)}\left(-\frac{\chi^{1 / 2}}{2 \sqrt{3}} \operatorname{coth}\left(\frac{\chi^{1 / 2}}{4 \sqrt{3}}\left(\xi+\xi_{0}\right)\right)+\frac{i \sqrt{3}(C(t)-\delta(t))}{2 A(t)^{1 / 2} B(t)^{1 / 2}}\right) \\
& \phi_{5}(\xi)=\frac{3(C(t)-\delta(t))}{2\left(\Phi_{1}\right)}\left(-\frac{1}{\left(\xi+\xi_{0}\right)}+\frac{i \sqrt{3}(C(t)-\delta(t))}{4 A(t)^{1 / 2} B(t)^{1 / 2}}\right)
\end{aligned}
$$

where

$$
\chi=\frac{64 a_{1}(t) a_{2}(t) B^{2}(t)-9(C(t)-\delta(t))^{2}}{A(t) B(t)}
$$


Hence, the Eq. (3.5) has solutions

$$
v_{i}(\xi)=-\frac{3(C(t)-\delta(t))}{4 B(t)}+a_{1}(t) \phi_{i}(\xi)+a_{2}(t) \phi_{i}(\xi)^{-1}, \quad i=1,2,3,4,5
$$

where $\phi_{i}(\xi), i=1,2,3,4,5$ are defined in (3.7)-(3.11) and $a_{1}(t), a_{2}(t)$ are arbitrary functions. According to (3.4) and (3.1), we established solutions of (1.1) in the original variables.

\subsection{Application of Exp-function method}

Following the Exp-function method, we assume that the solution of Eq. (3.5) can be represented in the form

$$
v(\xi)=\frac{a_{c} e^{c \xi}+\cdots+a_{d} e^{d \xi}}{b_{r} e^{r \xi}+\cdots+b_{s} e^{s \xi}}
$$

where $c, d, r$ and $s$ are positive integers to be determined later. It follows that

$$
v_{\xi \xi}=\frac{A_{0}}{B}+\frac{2\left(A_{0}\right)\left(B_{0}\right)}{(B)^{2}}
$$

and (3.5) takes the form

$$
\begin{aligned}
& \frac{1}{B}\left(2 A(t)\left(A_{0} B-2 \frac{\left(A_{1}\right)\left(B_{1}\right)}{(B)^{2}}+2 \frac{(A)\left(B_{1}\right)^{2}}{(B)^{3}}-\frac{(A)\left(B_{0}\right)}{(B)^{2}}\right)\right)(A)-A(t)\left(\frac{A_{1}}{B}-\frac{(A)\left(B_{1}\right)}{(B)^{2}}\right)^{2} \\
& -4 \frac{(\omega(t)+A(t)+\rho(t))(A)^{2}}{(B)^{2}}+4 \frac{(c(t)-\delta(t))(A)^{3}}{(B)^{3}}+4 \frac{B(t)(A)^{4}}{(B)^{4}}=0
\end{aligned}
$$

where

$$
\begin{array}{ll}
A=a_{1} \mathrm{e}^{\xi}+a_{0}+a_{-1} \mathrm{e}^{-\xi}, & B=b_{1} e^{\xi}+b_{0}+b_{-1} e^{-\xi}, \\
A_{0}=a_{1} e^{\xi}+a_{-1} e^{-\xi}, & B_{0}=b_{1} e^{\xi}+b_{-1} e^{-\xi}, \\
A_{1}=a_{1} e^{\xi}-a_{-1} e^{-\xi}, & B_{1}=b_{1} e^{\xi}-b_{-1} e^{-\xi} .
\end{array}
$$

Using these representations in (3.14), we arrive at the equation

$$
\begin{aligned}
& \left(-4 A(t) a_{1}^{2} b_{1}^{2}-4 \omega(t) a_{1}{ }^{2} b_{1}{ }^{2}-4 \rho(t) a_{1}{ }^{2} b_{1}{ }^{2}+4 B(t) a_{1}{ }^{4}+4 c(t) a_{1}{ }^{3} b_{1}-4 \delta(t) a_{1}{ }^{3} b_{1}\right) \\
& \quad \times\left(\mathrm{e}^{\xi}\right)^{8}+\cdots-4 A(t) a_{-1}{ }^{2} b_{-1}{ }^{2}-4 \omega(t) a_{-1}{ }^{2} b_{-1}{ }^{2}-4 \rho(t) a_{-1}{ }^{2} b_{-1}{ }^{2}+4 B(t) a_{-1}{ }^{3} \\
& \quad+4 c(t) a_{-1}{ }^{3} b_{-1}-4 \delta(t) a_{-1}{ }^{3} b_{-1}=0 .
\end{aligned}
$$

Equating to zero the coefficients at $e^{n x}$ leads to the system of algebraic equations for $a_{1}$, $a_{0}, a_{-1}, a_{1}, b_{0}, b_{-1}, b_{1}, \omega(t)$, and following the above considerations, we can discuss the following situations. 
Case 1.

$$
\begin{aligned}
& a_{1}=b_{1}=0, \quad a_{0}=\frac{(-c(t)+\delta(t)+\Psi) b_{0}}{2 B(t)}, \\
& a_{-1}=a_{-1}, \quad b_{-1}=\frac{(c(t)-\delta(t)+\Psi) a_{-1}}{2(\omega(t)+A(t)+\rho(t))},
\end{aligned}
$$

where

$$
\Psi=\sqrt{(\delta(t))^{2}-2 c(t) \delta(t)+4 B(t) A(t)+4 B(t) \omega(t)+4 B(t) \rho(t)+(c(t))^{2}} .
$$

Substituting these parameters into (3.13), we obtain the following solution of the Eq. (3.5):

$$
\begin{aligned}
v(\xi)= & \left(\frac{1}{2 B(t)}(-c(t)+\delta(t)+\Psi) b_{0}+a_{-1} \mathrm{e}^{-\xi}\right) \\
& \times\left(b_{0}+\frac{1}{2(\omega(t)+A(t)+\rho(t))}(c(t)-\delta(t)+\Psi) a_{-1} \mathrm{e}^{-\xi}\right)^{-1} .
\end{aligned}
$$

Therefore,

$$
\begin{aligned}
u(\xi)=\sqrt{v(\xi)}= & \left(\left(\frac{1}{2 B(t)}(-c(t)+\delta(t)+\Psi) b_{0}+a_{-1} \mathrm{e}^{-\xi}\right)\right. \\
& \left.\times\left(b_{0}+\frac{1}{2(\omega(t)+A(t)+\rho(t))}(c(t)-\delta(t)+\Psi) a_{-1} \mathrm{e}^{-\xi}\right)^{-1}\right)^{1 / 2}
\end{aligned}
$$

and

$$
\begin{aligned}
q(x, t)=e^{i \eta} u(\xi)=e^{i \eta} & \left(\frac{1}{2 B(t)}(-c(t)+\delta(t)+\Psi) b_{0}+a_{-1} \mathrm{e}^{-\xi}\right) \\
& \left.\times\left(b_{0}+\frac{1}{2(\omega(t)+A(t)+\rho(t))}(c(t)-\delta(t)+\Psi) a_{-1} \mathrm{e}^{-\xi}\right)^{-1}\right)^{1 / 2}
\end{aligned}
$$

with

$$
\eta=x+\int \omega(t) d t+\theta_{0}, \quad \xi=x+\int \lambda(t) d t+\xi_{0} .
$$

Case 2.

$$
\begin{array}{ll}
b_{1}=b_{1}, & a_{1}=\frac{(-c(t)+\delta(t)+\Theta) b_{1}}{2 B(t)}, \\
a_{-1}=a_{-1}, & b_{-1}=\frac{(c(t)-\delta(t)+\Theta) a_{-1}}{2(\omega(t)+A(t)+\rho(t))}, \\
a_{0}=a_{0}, & b_{0}=\frac{(c(t)-\delta(t)+\Theta) a_{0}}{2(\omega(t)+A(t)+\rho(t))},
\end{array}
$$


where

$$
\Theta=\sqrt{4 B(t) A(t)+4 B(t) \omega(t)+4 B(t) \rho(t)+(\delta(t))^{2}-2 \delta(t) c(t)+(c(t))^{2}}
$$

Substituting these parameters into (3.13), we obtain the following solution of the Eq. (3.5):

$$
\begin{aligned}
v(\xi)= & \left(\frac{1}{B(t)}(-c(t)+\delta(t)+\Theta) b_{1} \mathrm{e}^{\xi}+a_{0}+a_{-1} \mathrm{e}^{-\xi}\right) \\
& \times\left(b_{1} \mathrm{e}^{\xi}+\frac{1}{2(\omega(t)+A(t)+\rho(t))}(c(t)-\delta(t)+\Theta)\left(a_{0}+a_{-1}\right) \mathrm{e}^{-\xi}\right)^{-1} .
\end{aligned}
$$

Therefore,

$$
\begin{aligned}
u(\xi)= & \sqrt{v(\xi)} \\
= & \left(\left(\frac{1}{B(t)}(-c(t)+\delta(t)+\Theta) b_{1} \mathrm{e}^{\xi}+a_{0}+a_{-1} \mathrm{e}^{-\xi}\right)\right. \\
& \left.\times\left(b_{1} \mathrm{e}^{\xi}+\frac{1}{2(\omega(t)+A(t)+\rho(t))}(c(t)-\delta(t)+\Theta)\left(a_{0}+a_{-1}\right) \mathrm{e}^{-\xi}\right)^{-1}\right)^{1 / 2}
\end{aligned}
$$

and

$$
\begin{aligned}
q(x, t)= & e^{i \eta} u(\xi) \\
=e^{i \eta} & \left(\left(\frac{1}{B(t)}(-c(t)+\delta(t)+\Theta) b_{1} \mathrm{e}^{\xi}+a_{0}+a_{-1} \mathrm{e}^{-\xi}\right)\right. \\
& \left.\quad \times\left(b_{1} \mathrm{e}^{\xi}+\frac{1}{2(\omega(t)+A(t)+\rho(t))}(c(t)-\delta(t)+\Theta)\left(a_{0}+a_{-1}\right) \mathrm{e}^{-\xi}\right)^{-1}\right)^{1 / 2} .
\end{aligned}
$$

Case 3.

$$
\begin{array}{ll}
b_{-1}=b_{-1}, & a_{-1}=\frac{((-c(t)+\delta(t)+\Theta)) b_{-1}}{2 B(t)}, \\
a_{0}=a_{0}, & b_{0}=\frac{(c(t)-\delta(t)+\Theta) a_{0}}{2(\omega(t)+A(t)+\rho(t))} \\
b_{1}=a_{1}=0, & b_{-1}=\frac{(c(t)-\delta(t)+\Theta) a_{-1}}{2(\omega(t)+A(t)+\rho(t))}
\end{array}
$$

Substituting these parameters into (3.13), we obtain the following solution of the Eq. (3.5):

$$
\begin{aligned}
v(\xi)= & \left(a_{0}+\frac{1}{2 B(t)}(-c(t)+\delta(t)+\Theta) b_{-1} \mathrm{e}^{-\xi}\right) \\
& \times\left(b_{1} \mathrm{e}^{\xi}+\frac{1}{2(\omega(t)+A(t)+\rho(t))}(c(t)-\delta(t)+\Theta) a_{0}+b_{-1} \mathrm{e}^{-\xi}\right)^{-1} .
\end{aligned}
$$


Therefore,

$$
\begin{aligned}
u(\xi)= & \sqrt{v(\xi)} \\
= & \left(\left(a_{0}+\frac{1}{2 B(t)}(-c(t)+\delta(t)+\Theta) b_{-1} \mathrm{e}^{-\xi}\right)\right. \\
& \left.\times\left(b_{1} \mathrm{e}^{\xi}+\frac{1}{2(\omega(t)+A(t)+\rho(t))}(c(t)-\delta(t)+\Theta) a_{0}+b_{-1} \mathrm{e}^{-\xi}\right)^{-1}\right)^{1 / 2}
\end{aligned}
$$

and

$$
\begin{aligned}
q(x, t)= & e^{i \eta} u(\xi) \\
=e^{i \eta} & \left(\left(a_{0}+\frac{1}{2 B(t)}(-c(t)+\delta(t)+\Theta) b_{-1} \mathrm{e}^{-\xi}\right)\right. \\
& \left.\quad \times\left(b_{1} \mathrm{e}^{\xi}+\frac{1}{2(\omega(t)+A(t)+\rho(t))}(c(t)-\delta(t)+\Theta) a_{0}+b_{-1} \mathrm{e}^{-\xi}\right)^{-1}\right)^{1 / 2}
\end{aligned}
$$

with

$$
\eta=x+\int \omega(t) d t+\theta_{0}, \quad \xi=x+\int \lambda(t) d t+\xi_{0}
$$

\section{Conclusion}

We obtained exact solutions of the perturbed Gerdjikov-Ivanov equation with variable coefficients. Improved tanh-coth and Exp-function methods are used to establish various solutions, which can be used to determine new solutions of the classical perturbed GerdjikovIvanov equation with special sets of constant coefficients.

\section{Acknowledgements}

This work was supported by a research grant from the Amol University of Special Modern Technologies, Amol, Iran.

\section{References}

[1] H. Bulut, T.A. Sulaiman, H.M. Baskonus, H. Rezazadeh, M. Eslami and M. Mirzazadeh, Optical solitons and other solutions to the conformable space-time fractional Fokas-Lenells equation, Optik 172, 20-27 (2018).

[2] C.A. Gómez and A. Salas, Special symmetries to standard Riccati equations and applications, Appl. Math. Comput. 216(10), 3089-3096 (2010).

[3] D. Guo, S.F. Tian, X.B. Wang and T.T. Zhang, Dynamics of lump solutions, rogue wave solutions and traveling wave solutions for (3+1)-dimensional VC-BKP equation, East Asian J. Appl. Math. 9, 780-796 (2019). 
[4] M.G. Hafez, M.N. Alam and M.A. Akbar, Traveling wave solutions for some important coupled nonlinear physical models via the coupled Higgs equation and the Maccari system, J. King Saud Uni. Sci. 27(2), 105-112 (2015).

[5] J.H. He and X.H. Wu, Exp-function method for nonlinear wave equations, Chaos, Solit. Fract. 30(3), 700-708 (2006).

[6] L. Kaur and A.M. Wazwaz, Optical solitons for perturbed Gerdjikov-Ivanov equation, Optik 174, 447-451 (2018).

[7] A. Korkmaz, O.E. Hepson, K. Hosseini, H. Rezazadeh and M. Eslami, Sine-Gordon expansion method for exact solutions to conformable time fractional equations in RLW-class, J. King Saud Uni. Sci. https://doi.org/10.1016/j.jksus.2018.08.013, (2018).

[8] N.A. Kudryashov, One method for finding exact solutions of nonlinear differential equations, Commun. Nonlinear Sci. Numer. Simul. 17(6), 2248-2253 (2012).

[9] Z. Liu and C. Yang, The application of bifurcation method to a higher-order KdV equation, J. Math. Anal. Appl. 275(1), 1-12 (2002).

[10] W.X. Ma, Lump and interaction solutions to linear PDEs in (2+1)-dimensions via symbolic computation, Modern Phys. Lett. B 33, 1950457 (2019).

[11] W.X. Ma and B. Fuchssteiner, Explicit and exact solutions to a Kolmogorov-Petrovskii-Piskunov equation, Inter. J. Nonlinear Mech. 31, 329-338 (1996).

[12] W.X. Ma and J.H. Lee, A transformed rational function method and exact solutions to the $(3+1)$ dimensional Jimbo-Miwa equation, Chaos, Solits. Fract. 42, 1356-1363 (2009).

[13] W.X. Ma and L. Zhang, Lump solutions with higher-order rational dispersion relations, Pramana J. Phys. 94(1), 43 (2020).

[14] R.M. Miura, Korteweg-de Vries equation and generalizations. I. A remarkable explicit nonlinear transformation, J. Mathe. Phys. 9(8), 1202-1204 (1968).

[15] N. Nirmala, M.J. Vedan and B.V. Baby, Auto-Bäcklund transformation, Lax pairs and Painlevé property of a variable coefficient Korteweg-de Vries equation, J. Math. Phys. 27(11), 2640-2643 (1986).

[16] W.Q. Peng, S.F. Tian, X.B. Wang, T.T. Zhangand and Y. Fang, Riemann-Hilbert method and multisoliton solutions for three-component coupled nonlinear Schrödinger equations, J. Geom.Phys. 146, 103508 (2019).

[17] N. Raza, U. Afzal, A.R. Butt and H. Rezazadeh, Optical solitons in nematic liquid crystals with Kerr and parabolic law nonlinearities, Opti. Quan. Elec. 51(4), 107 (2019).

[18] N. Raza, M.R. Aslam and H. Rezazadeh, Analytical study of resonant optical solitons with variable coefficients in Kerr and non-Kerr law media, Opt. Quan. Elec. 51(2), 59 (2019).

[19] H. Rezazadeh, A. Korkmaz, M. Eslami and S.M. Mirhosseini-Alizamini, A large family of optical solutions to Kundu-Eckhaus model by a new auxiliary equation method, Opt. Quan. Elec. 51(3), 84 (2019).

[20] A.H. Salas, The Cole-Hopf transformation and improved tanh-coth method applied to the new integrable system (KdV6), Appl. Math. Comput. 204(2), 957-962 (2008).

[21] S.S. Singh, Dark and singular soliton solutions of perturbed Gerjikov-Ivanov equation via the first integral method, Inter. J. Phys. Research 6(2), 60-63 (2018).

[22] K.U. Tariq, M. Younis, H. Rezazadeh, S.T.R. Rizvi and M.S. Osman, Optical solitons with quadratic-cubic nonlinearity and fractional temporal evolution, Mod. Phys. Lett. B 32(26), 1850317 (2018).

[23] M. Wang, X. Li and J. Zhang, The $\left(G^{\prime} / G\right)$-expansion method and travelling wave solutions of nonlinear evolution equations in mathematical physics, Phys. Lett. A 372(4), 417-423 (2008).

[24] A.M. Wazwaz, The tanh-coth method for solitons and kink solutions for nonlinear parabolic equations, Appl. Math. Comp. 188(2), 1467-1475 (2007). 
[25] H. Yépez-Martínez, H. Rezazadeh, A. Souleymanou, S.P.T. Mukam, M. Eslami, V.K. Kuetche and A. Bekir, The extended modified method applied to optical solitons solutions in birefringent fibers with weak nonlocal nonlinearity and four wave mixing, Chin. J. Phys. 58, 137-150 (2019).

[26] H. Yilmaz, Exact solutions of the Gerdjikov-Ivanov equation using Darboux transformations, J. Nonlinear Math. Phys. 22(1), 32-46 (2015).

[27] L.D. Zhang, S.F. Tian, W.Q. Peng, T.T. Zhang and X.J. Yan, The dynamics of lump, lump-off and rogue wave solutions of $(2+1)$-dimensional Hirota-Satsuma-Ito equations, East Asian J. Appl. Math. 10(2), 243-255 (2020). 